\title{
Ist emphatischer Akzentwechsel bei expressiven (adjektivischen) Ausdrücken ein Hauptsatzphänomen des Deutschen?
}

\author{
André Meinunger \\ ZAS, Berlin
}

\section{Einleitung: Zum Phänomen des Akzentwechsels - Bekanntes und Neues}

Das im vorliegenden Artikel untersuchte Phänomen im Deutschen ist in der Literatur bisher quasi unentdeckt geblieben. Die einzige Ausnahme bildet der Beitrag von Berg (2008). Die Beobachtung ist folgende: Unter bestimmten Bedingungen, die mit Emphase zu tun haben, kann die lexikalisch festgelegte Betonung, also der Wortakzent, verschoben werden. Im Normalfall betrifft dieser Prozess nicht-native lexikalische Einheiten, denn die Akzentverschiebung passiert in der Regel von hinten nach vorn. Da deutsche Erbwörter initial-, also erstbetont, sind, ist das schwer möglich (jedoch s.u.). Fremdwörter, die auf den hinteren Silben betont sind, sind deshalb prädestiniert. Die meisten Beispiele kommen aus dem Bereich der Wortklasse Adjektiv: spektakulär, skandalös, sensationell, optimal, ideal, brutal, fulminant, perfekt, gigantisch. Im angedeuteten expressiven Gebrauch kann der Wortakzent von der letzten auf die erste Silbe wandern (angedeutet durch Großbuchstaben): SPEKtakulär, SKANdalös, SENsationell, OPtimal, Ideal, BRUtal, FULminant, PERfekt, GIgantisch. Es sind, wenn auch seltener, Substantive betroffen: Katastróphe $\rightarrow$ KAtastrophe, Phänomén $\rightarrow$ PHÄnomen, Mystérium $\rightarrow$ MYSterium. Berg nennt diesen Prozess „stress retraction“ (nach vor allem Gussenhoven 1983a,b), da die Betonung von rechts bzw. von hinten nach links bzw. nach vorn verschoben wird. Schematisch stellt er den häufigsten, Dreisilber betreffenden Fall folgendermaBen dar:

(1) $\quad--^{\prime}-\rightarrow^{\prime}---\quad$ (fulminant, optimal, skandalös, resolut, aktuell, fasziniert ...)

Für Berg ist diese Shiftrichtung die eigentliche. Betonungswechsel auf eine hintere Silbe (stress advancement) sei eine Art Ausnahme und gehorche besonderen, vorzugsweise morphologischen Regularitäten ${ }^{1}$. Dennoch lassen sich im Deutschen auch systematische Fälle von Links-rechts-Shift ausmachen. Eine

1 „We thus conclude that the infrequent group of stress advancements relies much more strongly on morphological structure than does the frequent group of stress retractions" (Berg 2008: 171). 
bisher gänzlich undiskutierte Entdeckung ist die Schwankung der Betonung bei nicht fremdwortartigen Adjektiven (und marginal auch bei den ihnen entsprechenden Substantiven). Interessant und einschlägig sind hier Adjektive, die mit der Silbe bzw. dem Morphem un- beginnen. Für die Klassiker Grimm (1878) und Paul (1920, 68: 22f.) ist un- (neben $u r$ - und erz-) quasi ein selbständiges Wort, denn Bildungen un- plus eine andere Wurzel bilden bei ihnen ein Kompositum. Dieses erklärt dann auch die Akzentverteilung, denn das Bestimmungswort trägt im Kompositum den prominenten Akzent: unfrei, unfertig, uneigennützig, ungezuckert. In den neueren Referenzwerken von einerseits Fleischer \& Barz (1992) und andererseits Erben (1993) gilt un- (wiederum neben ur-, erzund anderen) als Präfix - als eines, das den Akzent zu sich nimmt (wie die präpositionsähnlichen Elemente $a n-$, bei- ... zu-, auch ein-; oder die fremdsprachigen anti-, hyper-, ko-, pseudo- etc.). Nun kann scheinbar für eine Reihe von un-präfigierten Adjektiven keine eindeutige Betonung angegeben werden: unerhört, unglaublich, ungeheuerlich, ungemein, unsagbar, unsäglich, ungeheuer. Bei diesen und ähnlichen Adjektiven gibt die online-Dudenausgabe (www. duden. de) als erstes immer die Betonung wie zu erwarten auf $u n$ - an, also: $U N$ erhört, UNglaublich, UNgeheuerlich, UNheimlich usw. Oftmals existiert keine entsprechende un-lose Form: *erhört, *? glaublich, *? säglich (oder aber die unlose Form hängt mit der un-haltigen Form nicht systematisch-kompositional zusammen wie bei heimlich und unheimlich). Bei ebendiesen Adjektiven liefert der Online-Duden nun allerdings auch eine weitere Version, bei der die Hauptbetonung nicht auf die Erstsilbe fällt: unerHÖRT, unGLAUBlich, ungeHEUerlich, unHEIMlich etc. Beim Adjektiv unverschämt gibt die Grammatik nur die Erstbetonung an: UNverschämt. Mir scheint bei Emphase ebenso ein unverSCHÄMT möglich. Beim Adjektiv unendlich gibt die Grammatik interessanterweise nur die Version an, in der un- nicht prominent ist: unENDlich. Hier scheint die Initialbetonung allerdings auch möglich: UNendlich.

Ein oberflächlicher Blick auf die Adjektive mit wechselhafter Betonung, genauer gesagt auf die Adjektive, die über die spezielle Akzent-Alternative verfügen, (i) Wortakzent auf un- und bzw. oder (ii) Wortakzent auf einer hinteren Silbe, lässt schließen, dass Betonungswechsel an Emphase gebunden ist. Wichtig ist es, anzumerken, dass hier tatsächlich die neutrale Form diejenige mit Initialbetonung auf un- ist, was bedeutet, dass man es tatsächlich mit einer Form des Links-rechts-Shifts zu tun hat - und nicht umgekehrt. Nur solche Adjektive, die emphatisch gebraucht werden können, lassen die nicht-kanonische „Spät-“Betonung zu. Jene, die kaum bis unmöglich expressiv-emphatisch verwendet werden können, wie zum Beispiel die oben angeführten unfrei, unfertig, 
uneigennützig, ungezuckert, lassen die ,hintere“ Betonung nicht zu: *unFREI, *unFERtig, *unEIgennützig, *ungeZUCkert ${ }^{2}$.

Quasi parallel dazu verhalten sich partizipienverdächtige Adjektive wie ausgewechselt oder umgewandelt. Bei ausgewechselt gibt der Duden nur die initialbetonte Form AUSgewechselt; umgewandelt hat keinen eigenen Eintrag. Beim emphatischen Gebrauch kann die Betonung nach hinten verschoben werden.

(2) Nach der Pause spielte Hannover 96 wie umgeWANdelt. $(68)^{3}$

(3) Diese armen Menschen sind wie ausgeWECHselt. ${ }^{4}$

Dieser Shift - ebenfalls in Links-rechts-Manier - ist wiederum nur bei Emphase möglich. Es scheint also ein generelleres Phänomen zu sein als lediglich die umgekehrt-direktionale stress retraction. (Dennoch bleiben essentielle Fragen offen, denn diese Richtung scheint deutlich restringierter zu sein.)

Wie eingangs erwähnt, ist das Phänomen des Betonungswechsels an sich fast unbemerkt geblieben. Als erster scheint sich Gussenhoven den entsprechenden Daten im Niederländischen gewidmet zu haben. Von den zwei Arbeiten ist die eine eher phonologisch (1983a), die andere für die damalige Zeit äußerst modern experimentell-psycholinguistisch orientiert (1983b). In ebendieser wird der emphatisch-expressive Aspekt festgestellt. Gussenhoven spricht von ,,rhetorical retraction", also von einer rhetorischen Operation, oder im englischen Original von ,rhetorical device“. Derselbe, also ,rhetorical retraction ... seems particularly frequent in propagandist speech, as used for instance in commercials and political speeches [and is] frequent in the more informal news shows" (Gussenhoven 1983b: 604). Eine weitere Arbeit zum Thema und speziell zu deutschsprachigen Daten liefert der deutsche Anglist Berg. Als Phonologe ist dieser ebenfalls hauptsächlich an den lautgesetzlichen Aspekten interessiert. Ein Verdienst Bergs ist die Erstellung einer Belegsammlung authentischer ÄuBerungen (s.u. 2.1 und Fußnote 3). Die vorliegende Arbeit sieht sich als weiteren Beitrag zum Phänomen unter pragmatisch-semantisch-syntaktischer Perspektive.

2 Damit ist die Betonungsverschiebung zugunsten von Emphase gemeint: ungeZUCkert ist kontrastiv möglich - als Gegenüberstellung zu ungeSALzen etwa.

3 Die eingeklammerte Zahl nach einem Beispielsatz referiert auf die chronologische Nummer des authentischen Beleges aus Bergs handgeschriebener Liste. Weiter hinten treten diese Zahlen auch im Fließtext auf, z.B. als Beleg (91) oder Beleg (36), die konkreten Beispiele sind dabei kursiv gesetzt.

4 T. Vermes „Er ist wieder da“, Hörbuch in 6 CDs gelesen von C.M. Herbst: CD 4, Track 8. 


\section{Zum Verhältnis von Expressivität und Hauptsatzphänomenen}

Die Beschäftigung mit Emphase-Phänomenen hat in den letzten zehn Jahren vor allem seit und mit den Arbeiten von Potts (2005, 2007), die eine ganze Forschungsindustrie etabliert haben, einen enormen Aufschwung erlebt. Inzwischen erscheinen Überblickswerke und Handbücher zum Thema. Ein solches ist der 2013 erschienene, von Gärtner und Gutzmann herausgegebene Band Beyond expressives: Explorations in use-conditional meanings. Zu ebendiesem verfasste einer der beiden Herausgeber - Gutzmann - einen Überblicksartikel als Einführung in das Buch und gleichzeitig als Abriss der aktuellen Forschung. In diesem Beitrag stellt Gutzmann eine Liste aller Phänomene zusammen, die etwas mit Emphase und/oder Expressivität zu tun haben ${ }^{5}$. Diese sprachlichen Erscheinungen verfügen über eine wesentliche Komponente, die der rein wahrheitskonditionalen, klassisch-semantischen Ebene über- oder beigeordnet ist. Sie liefern einen Beitrag jenseits der Wahrheitsbedingungen; Gutzmann in seiner Sympathie mit dem Ansatz von Recanati (2004) nennt sie dementsprechend wie dieser nicht wahrheits- sondern gebrauchskonditional (use-conditional). (Hauptsächlich für das Deutsche einschlägige) Phänomene, die genannt und vorgestellt werden, sind:

- Pejorative Beifügung (dieser Idiot Hans)

- Expressive attributive Adjektive (dein verdammter Hund)

- Interjektionen (huch, verdammt)

- Expressiv gefärbte Ausdrücke (Köter, Arschloch)

- Modalpartikeln (eh, halt)

- Formale vs. vertrauliche Pronomen ( $d u$ vs. Sie im Deutschen)

- Ethischer Dativ (Komm mir ja nicht zu spät nachhause!)

- Fokusakzent

- Exklamative

- Verum-Fokus

- Appositive und Parenthesen

- Topikalisierung im Englischen (als Beispiel für Topikauszeichnung)

- Diminutive

- Nicht-flektierte Verben (dich in den Arm nehm)

Die Forschungssituation zu den einzelnen Phänomenen ist äußerst unterschiedlich, was vor allem auch dadurch zu erklären ist, dass die jeweilige Struktur

5 Gutzmann behauptet an keiner Stelle, seine Liste sei exhaustiv in Bezug auf die Literatursituation. Dennoch wird beim Leser das Gefühl geweckt, die in der bisherigen Forschung behandelten Phänomene, die Gutzmann auflistet, müssen quasi vollständig erfasst sein zumindest, was die Typen gebrauchskonditionaler Ausdrücke betrifft. 
unterschiedlich prominent im Sprachsystem ist. Außerdem ist nicht jedes Phänomen gleichsam interessant und wissenschaftlich herausfordernd. Ein Phänomen, das allerdings sowohl sehr häufig und charakteristisch als auch linguistisch interessant ist, sind Modalpartikeln im Deutschen. Diese tauchen auch immer wieder als Vertreter einer weiteren Kategorie auf: nämlich als sogenannte Hauptsatzphänomene (root clause phenomena; ab hier häufig abgekürzt als HP).

HP sind sprachliche Strukturen oder Eigenschaften, die nur in selbständigen (oder eben quasi selbständigen) Sätzen vorkommen. Die Pioniere der HPForschung sind Emonds (1970) und Hooper und Thompson (1973). Inzwischen gilt der Überblick von Heycock (2006) als Referenz. Davon lässt sich auch Jacobs (2014) inspirieren, wenn er (s)eine Liste für das Deutsche aufstellt. ${ }^{6}$

- Modalpartikeln

- Sprecherorientierte Adverbiale, Sprechaktadverbiale (ehrlich gesagt)

- Bestimmte Konjunktionaladverbiale (Obwohl Fritz immerhin schon das Zweite Staatsexamen hat, hat er sich noch nicht auf eine Lehrerstelle beworben.)

- I-Topikalisierung (Fritz ist mit Pauken und Trompeten durchs Examen gefallen, obwohl er /SO dumm

AUCH wieder nicht ist.)

- Sprechaktbezogene Anhängsel (Fritz hat schon das Zweite Staatsexamen, nicht wahr?)

- V2-Stellung (bzw. V1)

- Objektweglassung in Infinitiv-Aufforderungssätzen (Bitte (das Bild) nicht berühren!)

Modalpartikeln werden hier ganz prominent angegeben. Man könnte - nicht nur deshalb - meinen, dass es einen engen Zusammenhang zwischen gebrauchsfunktionalen Ausdrücken und Hauptsatzphänomenen gibt. Expressivität scheint einherzugehen mit Operationen, die auf Hauptsätze beschränkt sind. Das ist auch nachvollziehbar bei Sprechaktadverbialen, Exklamativität oder (emphatischer) Topikalisierung. Dennoch kann man leicht zeigen, dass viele der Gutzmannschen Beispiele für gebrauchsfunktionale Ausdrücke nicht wurzelsensitiv sind. Man findet sie also auch in typischen Nebensätzen. $\mathrm{Zu}$ denen gehören zentrale Adverbialsätze (z.B. Temporalsätze, ,echte“ Kausalsätze), restriktive Relativsätze oder beispielsweise Komplementsätze zu faktiven Prädikaten. (4) zeigt das mit einem expressiven Ausdruck im Temporalsatz und das authentische (5) im Vergleich zum nach dem Muster gebildeten (6) durch die Gleichwertigkeit im Hinblick auf den $d u$-Sie-Unterschied innerhalb eines faktiven $\mathrm{Ne}$ bensatzes.

6 Jacobs merkt ausdrücklich an, dass seine Liste keinen Anspruch auf Vollständigkeit erhebt. 
(4) Gerade als der Köter nochmals zum Schnappen ansetzt, stecke ich gewandt meine kleine Hand seitlich in sein Maul und drücke zu. (V. Guran: Von der Sünde zur Gnade, S. 95)

(5) So sehr es mich freute, dass Du bei den meisten Menschen so gut ankamst wie bei Evelines Mutter, so sehr ärgerte ich mich manchmal, weil ich mich des ... (I. Elsner: Briefe an einen ganz besonderen Hund, S. 68)

Genauso wie:

(6) So sehr es mich freute, dass Sie bei den meisten Menschen so gut ankamen wie bei Evelines Mutter, so sehr ärgerte ich mich manchmal, weil ich mich des ...

Die Frage, der hier nachgegangen werden soll, lautet: Ist der oben skizzierte Prozess des emphatischen Betonungswechsels ein HP oder ist er von der Kategorie „nicht-wurzelsensitive Operation“? Drei Betrachtungen sollen zur Beantwortung der Frage weitehelfen: 1) die Auswertung des Korpus von Thomas Berg; 2) die Auswertung der experimentellen Ergebnisse von Gussenhoven und 3) die Erstellung und Auswertung einer Befragung- bzw. Bewertungsstudie.

\section{Empirische Befunde}

\subsection{Bergs Ergebnisse - „Korpusbelege“}

In Berg (2008) wird auf eine vom Verfasser angelegte Datensammlung verwiesen. Diese hat mir der Autor auf Anfrage freundlicherweise überlassen. Sie besteht aus 158 niedergeschriebenen Beispielen - meist, aber nicht immer in Satzgröße. Diese ist allerdings zur Beantwortung unserer Frage entscheidend. Insofern fallen wahrscheinlich die meisten, jedenfalls ziemlich viele Äußerungen weg, denn aus einer Ellipse bzw. Einwort- oder Fragmentäußerung kann man nichts über den Status als HP ableiten (z.B. Beleg 110: Ein GRANdioser Sieg! Beleg 91: Die Mannschaft nun AGgressiver; Beleg 141: Scott Nicholls in einem FUriosen Schlussspurt; Beleg 36: ein REsoluter Einsatz von Günter Drews). Weiterhin kann und sollte man einige der von Berg gesammelten Beispiele ignorieren, die ziemlich eindeutig auf Performanzfehler zurückzuführen sind:

(7) Wenn Schweden ausgleicht, sind alle Bemühungen UMsonst gewesen; (120)

(8) Die beschäftigen ihn wochenWEIse, aber nicht länger. (23) 
Die akzeptabelsten, also die nachvollziehbarsten Fälle von Betonungswechsel findet man tatsächlich in Hauptsätzen.

(9) Jens Voigt hat offenbar SENsationell aufgetrumpft. (143)

(10) Wir werden das Center KOMplett umgestalten. (127)

(11) Die Fortbewegung der Schlange beruht auf einem einzigen, aber GEnialen Konstruktionsprinzip. (97)

(12) Die Geschichte ist nach wie vor sehr, sehr MYSteriös. (61)

(13) Da gibt es eine MASsive Intervention der Bundesregierung. (49)

Das spricht erst einmal für den Status als HP. Dennoch findet man in Bergs Sammlung auch Beispiele mit Betonungswechsel im Nebensatz. Es scheint allererdings, als könnte man diese potentiellen Gegenbeispiele „wegdiskutieren“. Beleg (19) in (14) kann als echter Fehler abgetan werden; hätte also denselben Status wie (23) oder (120). Diesen Schluss legt auch die Tatsache nahe, dass das Adjektiv industriell schwerlich als emphatisch gebraucht werden kann.

(14) Trotz der Tatsache, dass in der INdustriellen Welt ein Aufschwung stattfindet. (19)

(15) und (16) bzw. (37) und (33) enthalten betonungsgeshiftete Substantive; auch hier liegt ein zufälliger Fehler nahe. Die Sätze klingen nicht natürlich. Auch hier ist ein emphatischer Gebrauch quasi auszuschließen.

(15) Allgemein gilt, daß INteressenten sich an das Presseamt des deutschen Bundestages wenden können. (37)

(16) Der Eindruck, der hier entstanden ist, ist, dass es unterschiedliche sowjetische INterpretationen gibt. (33)

Außerdem gilt, dass HP nicht auf Wurzelsätze beschränkt sind, sondern, dass sie eben auch in hauptsatzähnlichen Nebensätzen auftreten können. Dazu zählen argumentrealisierende Sätze von Brückenverben (Verben des Sagens und Denkens (Reis 1997, Meinunger 2004 u.v.m.)) und sogenannte periphere Adverbialsätze (u.a. Frey 2011). Diese Prädikate erlauben zum Beispiel auch Verbzweit als wichtigste Eigenschaft (HP) unabhängiger deutscher Sätze. Interessanterweise lassen sich die Bergschen Belegsätze in Satzgefüge umformulieren, die den abhängigen Satz in Verbzweitgestalt beeinhalten. Das könnte ein Indiz sein, dass, falls die Sätze mit den Betonungswechseln doch akzeptabel sind, hauptsatzähnliche Strukturen vorliegen, bei denen man HP erwarten kann.

(17) Allgemein gilt, Interessenten können sich an das Presseamt des deutschen Bundestages wenden. (37') 
(18) Hier ist der Eindruck entstanden, es gibt / gebe unterschiedliche sowjetische Interessen. (33')

Eine ähnliche Erklärung kann für (19) bzw. (88) angeboten werden. Hier liegt mit dem als-Nebensatz ein Teilsatz vor, der ebenfalls hauptsatzhafte Verbstellung aufweist: Das finite Verb befindet sich in der linken Satzklammer. Dass dieses HP mit einem potentiell weiteren - dem emphatischen - Betonungswechsel kombiniert auftritt, sollte nicht verwundern. Dennoch gilt auch hier, dass die emphatische Interpretation des Adjektivs wieder sehr fragwürdig ist und das Beispiel als Performanzfehler gewertet werden kann.

(19) Es sieht so aus, als würden die Atomaren Kurzstreckenwaffen auf den Schrotthaufen wandern. (88)

Interessanter sind folgende Fälle:

(20) obwohl er das gestern noch KAtegorisch ausgeschlossen hatte (109)

(20) bzw. (109) mit der emphatischen Initialbetonung ist ebenfalls unproblematisch. Dieser Teilsatz - ein konzessiver Nebensatz - gilt als ein sogenannter peripherer Adverbialsatz. Diese können problemlos HP - zum Beispiel Modalpartikeln - aufweisen (Coniglio 2011, Frey 2011), wie der (109) nachgebildete Satz in (21) zeigt.

(21) obwohl er das gestern ja noch kategorisch ausgeschlossen hatte. $\left(109^{\prime}\right)$

In (22) bzw. (54) liegt Betonungsshift innerhalb eines infiniten Nebensatzes vor.

(22) Die Städteverwaltung hatte versprochen, die Protestaktionen als LEgal anzuerkennen. (54)

Es scheint plausibel, dass der Betonungswechsel hier nicht emphatisch, sondern kontrastiv gemeint ist. Das kann allein aufgrund des einzelnen Gesamtsatzes nicht entschieden werden, aber die Vermutung liegt nahe, dass hier eine Reaktion auf eine Aussage, in der das Wort illegal vorkommt, getroffen wird. Im Kontrast zu illegal wird legal dann auf seiner ersten Silbe betont. Somit wäre (20) bzw. (109) als Beispiel bei der Beantwortung der Frage „HP oder nicht?“ nicht einschlägig.

Zusammenfassend kann man aus Bergs Arbeiten nicht eindeutig extrapolieren, ob der einschlägige Betonungswechsel wurzelsensitiv ist oder nicht. Allerdings geht die Auswertung doch stark in die Richtung, dass es sich um ein HP handelt. 


\subsection{Gussenhovens Ergebnisse - Resultate des Elizitierens}

Gussenhoven weist den expressiven oder, wie er es nennt, rhetorischen Effekt von Betonungswechseln (stress retraction) im psycholingustischen Experiment nach. Dazu werden Texte mit einer auffordernden Botschaft erzeugt, die dann von Testpersonen vorgelesen werden müssen. Die Texte enthalten eine Reihe von Adjektiven, die expressiv verwendet werden können und die von ihrer phonologischen Struktur her für einen Betonungswechsel geeignet sind. Der eine ausgewertete Text ist ein politischer Appell, in dem die Adressaten aufgerufen werden, an einer Protestaktion teilzunehmen. Der andere ist ein Werbetext, der zum Kauf eines Zahnpflegemittels animieren soll. Verschiedene Gruppen werden instruiert, aus welcher Perspektive sie den Text vorlesen sollen. So sollen einige Probanden den Text als Testsprecher lesen, damit ein angeblich neues Spracherkennungssystem trainiert und getestet werden kann, das gesprochene Sprache in verschriftete umwandelt. Eine andere Gruppe soll den Text so lesen, dass er möglichst effektiv bei den Adressaten ankommt, wieder eine andere Gruppe soll sich in die Lage eines PR-Teamchefs versetzen, der für den Text verantwortlich ist, ihn aber in der jetzigen Form für ungeeignet und stark verbesserungswürdig hält. (Es gibt noch eine weitere Gruppe.) Es ist gut nachzuvollziehen, dass die Leser jeweils unterschiedlich zum Textinhalt eingestellt sind. So sind die der ersten Gruppe wohl neutral, die der zweiten positiv, engagiert - die der dritten Gruppe reserviert oder negativ eingestellt. Gussenhoven kann zeigen, dass diejenigen, die hinter der Botschaft des Textes stehen (sollen), signifikant häufiger Betonungswechsel praktizieren. Leider sind die Texte wenig aussagekräftig, was die Typisierung von Stress-Shift als HP betrifft. Bis auf eine Ausnahme finden sich sämtliche potentiell akzentverschiebenden Adjektive in Hauptsätzen. Das könnte wieder eine Indiz für den HP-Status sein; allerdings kein starkes. Die einzige Ausnahme mit einem Adjektiv (bzw. zwei Adjektiven) in einem abhängigen Satz platziert diese(s) innerhalb eines restriktiven Relativsatzes. Der dem niederländischen Originalbeispiel entsprechende deutsche Satz würde lauten:

(23) Sesal ist nicht weniger als eine geniale Erfindung, die auf einfache, aber radikale Weise Schluss macht mit dem, was die Zahnmedizin als eine immanente Volkskrankheit betrachten musste: Karies.

Signifikant häufig wurde radikaal ,radikal ' geshiftet. Das ist aber wenig verwunderlich, liegt hier doch Kontrast zu eenvoudig ,einfach“ vor. Damit wird hier das Muster LEgal - ILlegal reproduziert. Allerdings wurde weitaus häufiger strukturel(e) (hier übersetzt mit immanent) geshiftet. Das Adjektiv war das wechselfreudigste im ganzen Werbesample überhaupt. Dieser Befund sollte dann zu denken geben, denn dieses Wort ist das am tiefsten eingebettete: 
Es findet sich innerhalb eines restriktiven Relativsatzes, der seinerseits in einen restriktiven Relativsatz eingebettet ist ${ }^{7}$. Relativsätze weisen aber ziemlich problemlos HP auf. Als Fazit zeichnet sich Betonungswechsel wiederum als HP ab.

\subsection{Neuere Ergebnisse - Interpretation der Resultate aus einer Informanten-Befragung}

In einer kleineren Fragestudie habe ich einen Beurteilungsbogen erstellt, der vierzehn Sätze enthält. Alle Sätze waren (bzw. sind) minimal komplex; das heißt, sie bestehen aus einem Hauptsatz, in den jeweils ein Nebensatz eingebaut ist. Dieser Nebensatz enthält ein expressives Adjektiv, dessen emphatische Betonung durch Großschreibung angedeutet ist. Die Probanden wurde angehalten, auf einer Skala von 1 (vollkommen akzeptabel) bis 5 (unmöglich) zu beurteilen, wie gut die Sätze klingen (könnten). Die Versuchspersonen waren 17 Kollegen vom ZAS (Berlin) und 8 Studierende der Universität Leipzig, also insgesamt 25 linguistisch (vor-)gebildete Muttersprachler(innen). Erwartungsgemäß wurden zwei Sätze als die besten bewertet, die Nebensätze enthalten, die HP lizenzieren (in dem Fall realisiert durch die Verbzweitsellung). Das waren (24) mit der Durchschnittbewertung 1,18 und (25) mit der von 1,39.

(24) Claudia meint, Kerstin sei die Ideale Kandidatin. (1,18)

(25) Peter meint, der Auftritt im LK-Club gestern war SENsationell. $(1,39)$

Als schlechtester, d.h. quasi als unakzeptabel $(3,9)$ wurde $(26)$ bewertet.

(26) Wir bestreiten, dass die Tests und Versuche OPtimal gelaufen sind. $(3,94)$

Ähnlich schlecht war (27) mit der Bewertung 3,4.

7 Auf das Deutsche lässt sich das Datum schwerlich übertragen. Der Versuch, den niederländischen Originaltext zu übersetzen, gelingt durch das Adjektiv immanent nur mäßig. Gussenhoven, der seinen eigenen Probe-Text für den Aufsatz ins Englische übersetzt, lässt einen adjektivischen Modifikator gänzlich weg, was die Frage aufwirft, welchen Beitrag - semantisch und expressiv - das Adjektiv strukturel letztendlich hat.

Niederländisches Original: Sesal is zonder meer een geniale vinding, die op eenvoudige, maar radikale wijze een einde maakt aan wat de medische professie tot op heden als een strukturele volksziekte heeft moeten beschouwen: tandbederf!

Englische Übersetzung durch den Autor: Sesal is nothing less than a brilliant discovery, which in a simple but radical manner puts a stop to what the medical profession has so far had to consider an endemic disease: tooth decay! 
(27) Peter hat sich gefreut, dass der Auftritt im LK-Club gestern SENsationell war. $(3,4)$

Dieses Bewertungsverhalten ist zu erwarten, wenn man den Betonungswechsel als HP begreift: bestreiten und sich freuen als faktive Verben lassen keine HP zu. Dennoch zeigen die Befragungsergebnisse, dass für den emphatischen Betonungswechsel keine Wurzelsensitivität besteht. Als nahezu genauso perfekt wie (24) und (25) wurden die Sätze (28) und (29) beurteilt:

(28) Hans kotzt es an, dass sein Nachbar PERmanent Krach macht. $(1,21)$

(29) Sie fand es gut, dass er OPtimal vorbereitet kam und KONstant bei der Sache war. $(1,40)$

Hier findet sich die emphatische Akzentuierung in einer Umgebung, die extrem HP-phob ist: innerhalb von Argumentsätzen zu faktiven Ausdrücken, in einem Fall sogar innerhalb eines Subjektsatzes.

Auch in zentralen Adverbialsätzen wurden Betonungswechsel von vielen Probanden als einigermaßen akzeptabel $(2,4$ bzw. 2,31) beurteilt.

(30) Klaus war im Klub, als Peter den PERfekten Auftritt hingelegt hat. $(2,40)$

(31) Weil Heike Raucher KAtegorisch ablehnt, wird Udo kaum ne Chance haben. $(2,31)^{8}$

\section{Ergebnis}

Insofern kann man ganz eindeutig schließen, dass die emphatische Operation Betonungswechsel (stress shift) nicht wurzelsensitiv ist. Sie ist also kein Hauptsatzphänomen. Damit hat sie einen Expressivitäts-Status vergleichbar mit Diminutivbildung, Siezen vs. Duzen oder dem Beitrag expressiver Ausdrücke wie Köter, Töle, Karre, verdammt, verflucht, beschissen, sahne-, hammer- u. ̈̈.

\section{Literatur}

Berg, Thomas (2008). Emphatic stress shift in German. Zeitschrift für Sprachwissenschaft 27(2): $165-187$.

Coniglio, Marco (2011). Die Syntax der deutschen Modalpartikeln: Ihre Distribution und Lizenzierung in Haupt- und Nebensätzen (= Studia grammatica 73). Berlin: Akademie Verlag.

8 Warum diese adverbialsatzhaltigen Sätze doch markierter sind als diejenigen mit Argumentsätzen - also als schlechter beurteilt werden, ist nicht klar. Möglichwerweise ist das Sample an Sätzen nicht groß genug, um zu generalisieren. Entscheidend ist jedoch, dass sie als relativ gut empfunden werden. 
Emonds, Joseph (1970). Root and structure preserving transformations. Dissertation, Cambridge MIT.

Erben, Johannes (1993). Einführung in die deutsche Wortbildungslehre (= Grundlagen der Germanistik 17). Berlin: Erich Schmidt.

Fleischer, Wolfgang \& Irmhild Barz (1992). Wortbildung der deutschen Gegenwartssprache. Tübingen: Niemeyer

Frey, Werner (2011). Peripheral adverbial clauses, their licensing and the prefield in German. In: E. Breindl, G. Ferraresi \& A. Volodina (Hrsg.). Satzverknüpfung - Zur Interaktion von Form, Bedeutung und Diskursfunktion. Berlin: De Gruyter, 41-77.

Grimm, Jacob (1878). Deutsche Grammatik. T. V. Neuer vermehrter Abdruck. Besorgt durch W. Scherer, Gütersloh.

Gussenhoven, Carlos (1983a). Focus, mode and the nucleus. Journal of Linguistics 19: 377417.

Gussenhoven, Carlos (1983b). Stress shift in Dutch as a rhetorical device. Journal of Linguistics 21: 603-619.

Gutzmann, Daniel (2013). Expressives and Beyond: An introduction to varieties of useconditional meaning. In: D. Gutzmann \& H.-M. Gärtner (Hrsg.). Beyond expressives: Explorations in use-conditional meaning. Leiden: Brill, 1-55.

Heycock, Caroline (2006). Embedded root phenomena. In: M. Everaert \& H. van Riemsdijk (Hrsg.). The Blackwell Companion to Syntax. Band 2. Oxford: Blackwell, Chapter 23: 174-209.

Hooper, Joan \& Sandra Thompson (1973). On the Applicability of Root Transformations. Linguistic Inquiry 4: 465-497.

Jacobs, Joachim (2014). Haben Nebensätze Satzmodus? Vortrag Wuppertaler Linguistisches Forum, 3.7.2014.

Meinunger, André (2004). Verb position, verbal mood and the anchoring (potential) of sentences. In: H. Lohnstein \& S. Trissler (Hrsg.). Syntax and Semantics of the Left Periphery. Mouton de Gruyter: Berlin, 313-341.

Paul, Hermann (1920/1968). Deutsche Grammatik. Band V, T. V: Wortbildungslehre. Tübingen: Max Niemeyer.

Potts, Christopher (2004). The Logic of Conventional Implicatures (= Oxford Studies in Theoretical Linguistics 7). Oxford: Oxford University Press.

Potts, Christopher (2007). The expressive dimension. Theoretical Linguistics 33: 165-197. http: //people.umass . edu/potts/papers/potts-expressives06.pdf.

Recanati, François (2004). Pragmatics and Semantics. In: L. Horn \& G. Ward (Hrsg.). The Handbook of Pragmatics. Oxford: Blackwell, 442-462.

Reis, Marga (1997). Zum syntaktischen Status unselbständiger Verbzweit-Sätze. In: C. Dürscheid, K.-H. Ramers \& M. Schwarz (Hrsg.). Sprache im Fokus. Tübingen: Niemeyer, 121-144. 